\title{
Silver coordination compounds with antimicrobial properties
}

\author{
Katharina M. Fromm*
}

\begin{abstract}
Silver and its compounds have long been known to possess antimicrobial properties. We report here on our observations in this field of research, namely on silver coordination compounds, and in particular polymers, which can be used in the medical field. An overview of the structural diversity of coordination compounds with a particular class of organic ligands is given, together with their properties, with a special focus on antimicrobial activity, solubility and light stability. Copyright $\odot 2013$ John Wiley \& Sons, Ltd.
\end{abstract}

Keywords: silver; coordination compounds; coordination polymers; antimicrobial properties; solubility; light stability

\section{Introduction}

Silver has been known for centuries to possess beneficial properties when it comes to the disinfection of water, for example. ${ }^{[1]}$ Hence the tradition of people throwing a coin into the water of a 'wishing well' is based on the fact that at one time these were silver coins, and drinking from such a well kept people healthier compared to drinking from other water sources. Alexander the Great kept his drinking water in silver vessels.

In modern times, $1 \%$ silver nitrate solution is used as an ointment for the treatment of newborn babies' eyes in order to prevent infections which might occur during birth. It has a similar efficiency in its antimicrobial properties to tetracycline, erythromycin or other alternative antibiotics that may be used. ${ }^{[2]}$ Another frequently used substance is silver sulfadiazine, which is the active component in many topical creams for burn wounds, and prevention or treatment of bacterial or fungal infections. ${ }^{[3]}$

Although silver and its compounds were known as effective disinfectants, they were often forgotten or replaced when antibiotics were discovered and brought to the market in the 1940s. Now that bacteria are becoming increasingly resistant to these antibiotics, silver is again back 'on the stage' as a possible player to combat even multi-resistant bacteria. ${ }^{[4]}$ Its detailed mechanism of action is yet unclear at the molecular level, as there is not just one target for silver in a cell. It is said to interact with the cell membrane, proteins, enzymes and even DNA. ${ }^{[5]}$ This large field of action, with numerous targets, makes studying its mechanism of action a big challenge on one hand, while on the other it seems less likely for cells to develop resistance. For the more interested reader on the applications of silver in medicine, the author recommends a recent review article, also taking also into account silver nanoparticles. ${ }^{[6]}$ The antimicrobial properties of nanoparticles in general have been highlighted in another article. ${ }^{[7]}$ Those interested in coordination polymers may refer to a review on the subject which focuses in particular on silver coordination polymers. ${ }^{[8]}$

In the following, we will describe in short the coordination compounds of silver which we obtained with a class of biocompatible ligands, namely derivatives of short polyethylene glycol (PEG), functionalized at both ends with (iso-)nicotinic acid groups. Their structure families will be highlighted with some examples, along with their properties, before overviewing their antimicrobial properties.

\section{Silver Coordination Compounds}

In the Fromm group, we have developed a class of flexible, easily upscalable and biocompatible ligands (Scheme 1) derived from short oligomers of polyethylene glycol, derived at the end groups with either isonicotinic or nicotinic acid. All ligands were tested in vitro for their biocompatibility against mouse fibroblast cells, showing no effect whatsoever on vitality and growth.

Regarding the nomenclature for our family of ligands, Li stands for the bis-isonicotinic derivatives, Ln for the bis-nicotinic acid compounds, and an additional number indicates the length of the ligand. Thus, for instance, Ln2 is the diethylene glycol derivative with nicotinic acid moieties at both ends.

For a number of ligands, we were able to determine their structure in the solid state. We have shown recently ${ }^{[9]}$ that although the $\mathrm{C}-\mathrm{C}$ bond within the glycols is in principle very flexible, the short ligands in particular seem to have preferential conformations in the solid state. Indeed, the ligands Li1, Li3, Ln2 and Ln4 adopt an overall 'S'-shape with an anti conformation at least at one ethylene group. Conversely, the ligands Li2, Li4, Ln1 and Ln3 prefer rather a more or less flat ' $U$ '-shape in the solid state (Scheme 2). As discussed earlier, we believe this is due to the overall dipole moment of the ligand molecule and the packing possibilities. ${ }^{[9]}$ Model calculations of the solid-state structures of these ligands are still missing, but would hopefully provide insight into understanding and predicting such structures in the future. Indeed, as we will show below, the ligands most often maintain their conformation upon coordinating to silver ions to form new solid-state structures.

\footnotetext{
* Correspondence to: Katharina M. Fromm, Department of Chemistry, University of Fribourg, Chemin du Musée 9, 1700 Fribourg, Switzerland. Email: Katharina. fromm@unifr.ch
}

Department of Chemistry, University of Fribourg, 1700 Fribourg, Switzerland 


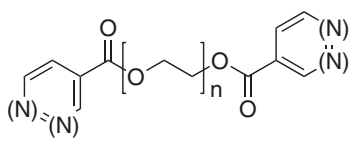

Scheme 1. Family of ligands used for the construction of silver coordination compounds; $n=1-4,6,8,10,12,>100$.

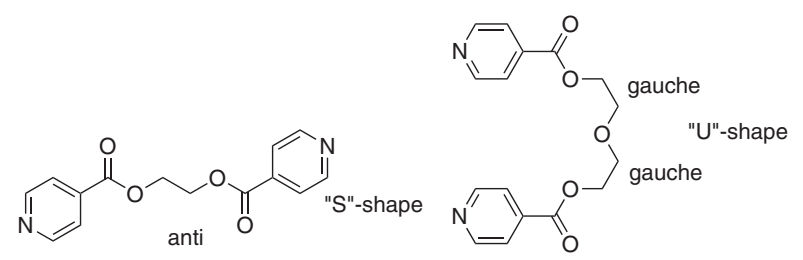

Scheme 2. Ligand conformations in the solid state for Li1 and Li2.

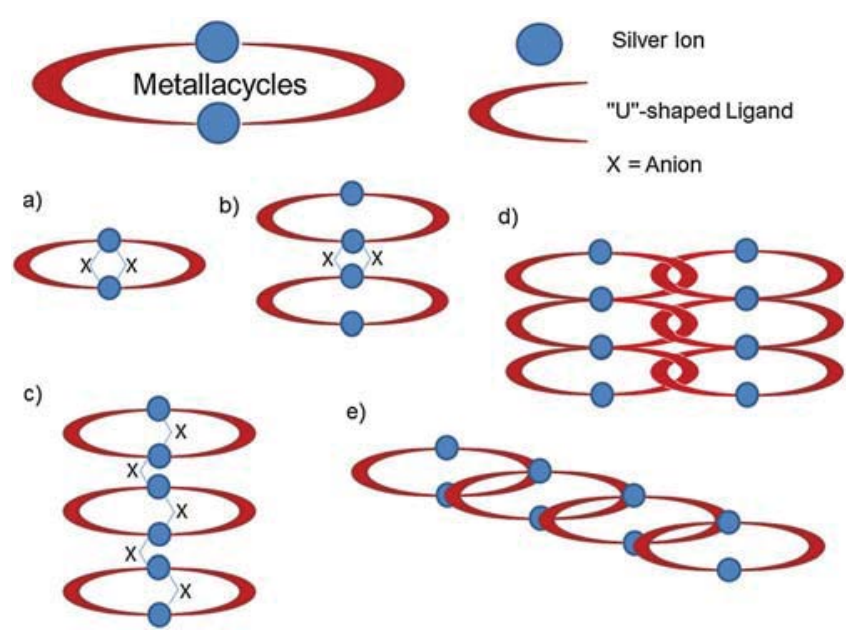

Scheme 3. Different ways of arranging silver metallacycles: (a) isolated with intra-ring bridging anions; (b) connected by bridging anions; (c) inter- as well as intra-ring connected; (d) by polycatenation when $L$ is in excess; (e) stacking of rings for larger ligands.

In the following, the results are presented as a function of the dimensionality of the compounds in the solid state. All compounds have been identified via single-crystal structure determination, while the bulk material was compared with the crystals by powder X-ray diffraction to confirm identity.

\section{Metallacylic ('Ring') Compounds}

From a thermodynamic viewpoint, in particular entropy, and starting from the same building blocks, the formation of metallacyclic species should be preferred over the construction of polymeric structures if the geometry of the ligands allows cyclic structures. Indeed, the ' $U$ '-shaped ligands of our ligand series seem to maintain their preferred conformation in the solid state after coordination to silver ions. In the case of silver salts AgX $\left(\mathrm{X}=\mathrm{NO}_{3}{ }^{-}, \mathrm{PF}_{6}{ }^{-}, \mathrm{ClO}_{4}{ }^{-}\right.$or $\left.\mathrm{CF}_{3} \mathrm{SO}_{3}{ }^{-}\right)$, the ligand $\mathrm{Li} 2$, for instance, yields a panoply of different ring systems of the general composition $[\mathrm{Ag}(\mathrm{Li} 2)]_{2} \mathrm{X}_{2}$ when reacted in a 1:1 ratio, independently of the solvent. ${ }^{[10]}$ The latter only has an influence on the role of the anions, which can act as bridging ligands across two silver ions of the same metallacycle, or else connect two metal ions of two neighbor units (Scheme 3). For the very versatile nitrate ion, we were able to observe all three possibilities, both anions bridging within the metallacycle, both between two neighbor metallacycles, or else one within, the second between the metallacycles. This was influenced by the solvent and the crystallization conditions. We have shown that the bridging role of the anions changes and influences the intra- as well as interring $\mathrm{Ag}-\mathrm{Ag}$ distances, ranging from $\sim 2.8$ to $3.3 \AA$ in the solid state. ${ }^{[10]}$ This variety of tunable metal-metal distance leads to different light sensitivities of the various macrocyclic compounds. Hence we have shown earlier that compounds based on strongly coordinating anions, which act like clamps between two metal ions, have shorter $\mathrm{Ag}-\mathrm{Ag}$ distances and are more light- sensitive than the metallacycles, with less strong coordinating anions and longer $\mathrm{Ag}-\mathrm{Ag}$ distances. Thus silver nanoparticles are more easily formed when the initial metal-metal distances are short. ${ }^{[10,11]}$ This aspect of structure tuning can be important in the design of medically usable compounds, as medical doctors are usually reluctant to use compounds which change aspect with time or upon irradiation.

Li2, due to its strong ' $U$ '-shape, yields almost exclusively metallacycles when reacted in a 1:1 ratio with the above-mentioned silver salts, except in one case, where the ligand is slightly in excess versus the metal ion. We were able to show previously that $[\mathrm{Ag}(\mathrm{Li} 2)]^{+}$is the predominant species in solution for a $1: 1$ reaction leading to the metallacycles, whereas a slight excess of ligand induces the formation of $\left[\mathrm{Ag}(\mathrm{Li} 2)_{2}\right]^{+}$ions, which in turn favor the formation of a one-dimensional helical chain, as observed for the example of $\mathrm{AgClO}_{4}{ }^{[12]}$ Thus the compound $\left\{\left[\mathrm{Ag}(\mathrm{Li2} 2) \mathrm{ClO}_{4}\right\}_{n}\right.$ can have $n=2$, forming metallacycles, or $n=\infty$ for the helix, the two compounds being isomers (Fig. 1).

Adding more Li2 in order to achieve a ratio of 2:1 with the $\mathrm{AgPF}_{6}$ salt, the initial metallacycles (i) are bridged by the additional ligands and (ii) interpenetrate such as to form a twodimensional, polycatenated structure (Fig. 2 and Scheme 3d). ${ }^{[13]}$ This structure is still unique to the best knowledge of the author, building a very robust framework.

With the larger ' $U$ '-shaped ligands, larger ring compounds can be obtained as well. ${ }^{[9]}$ For instance, the ligand Li4 forms large metallacycles by coordination of two ligands to two silver ions. The rings are, however, not isolated or simply bridged by anions, as for Li2, but the cyclic entities stack offset on top of each other. The offset is such that the $O$ atoms of the polyether chain of one ligand molecule of one ring can coordinate to one silver ion of the ring below. On the other hand, the polyether chain of the second ligand in the ring binds to one silver ion of the next ring above. Hence an overall stepped stacking of rings is observed (Scheme 3e) in particular for weakly

Figure 1. A ring (left) and a helix (right) - the two isomers for $\left\{[\mathrm{Ag}(\mathrm{Li2})] \mathrm{ClO}_{4}\right]_{n}{ }^{[12]}$ 


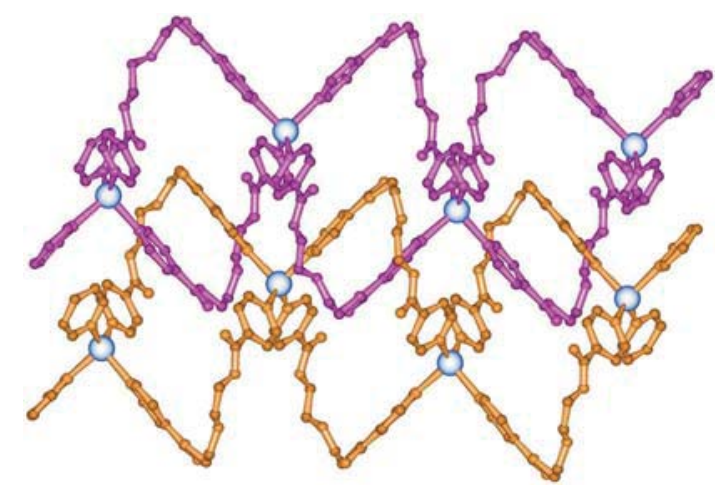

Figure 2. Polycatenated framework of $\left[\mathrm{Ag}(\mathrm{Li} 2)_{2}\left(\mathrm{PF}_{6}\right)\right]_{n}(\mathrm{H}$ atoms and anions omitted for clarity). ${ }^{[13]}$

coordinating anions such as the triflate $\mathrm{CF}_{3} \mathrm{SO}_{3}{ }^{-\left[{ }^{[9]}\right.}$ Although Ln4 belongs to the ligand group forming an ' $\mathrm{S}$ '-shape rather than a ' $\mathrm{U}$ '-shape, and with the two $\mathrm{N}$ atoms pointing in opposite directions, it is flexible enough also to form metallacycles of the type $[\mathrm{Ag}(\mathrm{Ln} 4)]_{2}{ }^{2+}$ with nitrate as a strongly binding counter ion. However, due to the strong coordinating power of nitrate, the rings are isolated and the polyether units do not interact with any metal ion (Fig. 3a). This can be changed by increasing the silver salt ratio to 2:1 versus the ligand, yielding $\left[\mathrm{Ag}_{2}(\mathrm{Ln} 4)\left(\mathrm{NO}_{3}\right)_{2}\right]_{2} .{ }^{\left[{ }^{[9]}\right.}$ Then, nitrate acts as a bridging ligand between the rings, and the additional silver ions are also coordinated by the polyether moieties of the ligands (Fig. 3b).

\section{One-Dimensional Coordination Polymers: Chains, Helices and Double Helices}

The ligand Li1 arranges into quasi-flat molecules with an 'S'-shape, both $\mathrm{N}$-donor atoms pointing at $180^{\circ}$ to each other. Despite its principal flexibility about the $\mathrm{C}-\mathrm{C}$ bond, it maintains this anti conformation in the large majority of its coordination compounds with silver. The silver ion preferring the coordination number 2, and with a quasi-linear ligand as linker, the generation of simple chains can be anticipated. Indeed, we could show that such structures are obtained and, depending on the counter ion, can lead to structural isomerism and polymorphism. ${ }^{14-19}$ Indeed, for the compound $\left[\mathrm{Ag}(\mathrm{Li} 1) \mathrm{NO}_{3}\right]_{n}$, two polymorphs based on simple chains and one isomer based on pairs of chains are found. Co-crystallizing solvent molecules lead furthermore to solvates of this compound. Changing the anion leads to similar structure a)

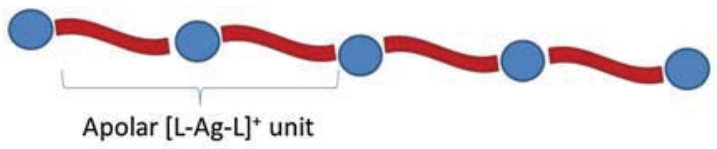

b)

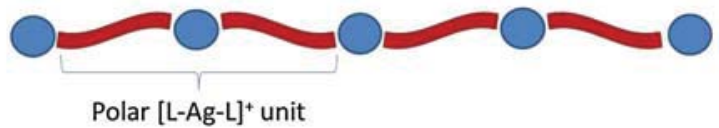

c)

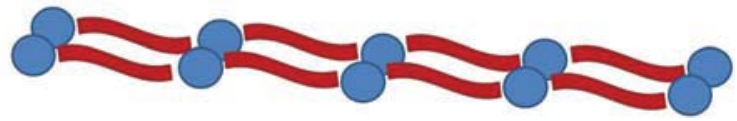

Scheme 4. Schematic representation of the polymorphs (a) and b)) of $\left[\mathrm{Ag}(\mathrm{Li1}) \mathrm{NO}_{3}\right]_{n}$, and a structural isomer of the same composition (c).

types as shown in Scheme 4. Only in one case so far does the ligand Li1 adopt a gauche conformation when coordinating to silver ions (it is also found to adopt both anti and gauche conformation upon binding to $\mathrm{Cu}(\mathrm{l})$ ), but even so the formed chains are rather flat.

Upon changing the position of the N-donor, using ligand Ln1, which has a preferred gauche conformation in the solid state, its coordination polymers with silver form single helical structures (Fig. 4)..$^{[9,20]}$ In such cases, the metal ion is usually coordinated by the anions, e.g. $\mathrm{NO}_{3}{ }^{-}$or $\mathrm{CF}_{3} \mathrm{SO}_{3}{ }^{-}$.

As longer ligands are used, their degree of flexibility increases, allowing the ligand to adopt different conformations upon coordination to metal ions. In the case of silver ions, the (quasi-)linear motif $\mathrm{N}-\mathrm{Ag}-\mathrm{N}$ is maintained, but in addition the O-atoms of one ligand of a chain wrap around a silver ion of a second chain as well, leading thus to the formation of a double helical motif. In the case of $\mathrm{Li} 3$, the ligand can bind with two $\mathrm{O}$ atoms to the metal ion, and together with the $\mathrm{O}$ atom of the anion $\mathrm{NO}_{3}{ }^{-}$or $\mathrm{CF}_{3} \mathrm{SO}_{3}{ }^{-}$the $\mathrm{O}$ atoms form the equatorial plane of a distorted trigonal bipyramid around silver. ${ }^{[9]}$ The $\mathrm{N}$ atoms of two different ligands occupy the axial position; hence three different ligands are coordinating to a silver ion at once (Fig. 5a).

The ligand Ln4, just as Ln6, is also able to form double-helical structures with silver salts of non- or weakly coordinating anions like $\mathrm{BF}_{4}{ }^{-}$or $\mathrm{PF}_{6}{ }^{-}$, respectively, as has been described by Hosseini et al. (Fig. 5b). ${ }^{[21]}$

Our so far studies have been limited to the ligand series Li1-Li4 and $\operatorname{Ln} 1-\operatorname{Ln} 4,{ }^{[9,14-19]}$ while a paper on longer ligands is currently in preparation. ${ }^{[22]}$ It is clear, however, that the longer the ligand, the more flexible are the ligands, allowing for more structural diversity, including interpenetrated structures.

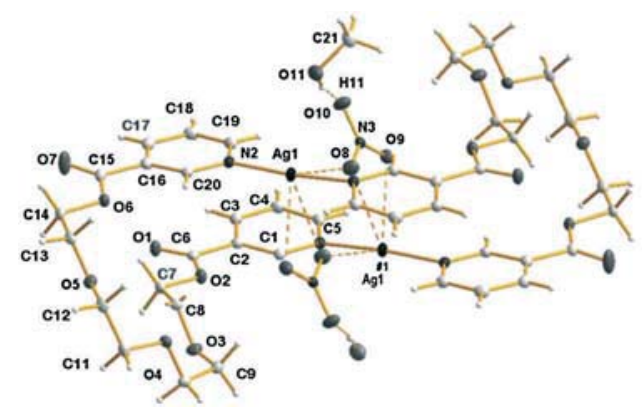

a)

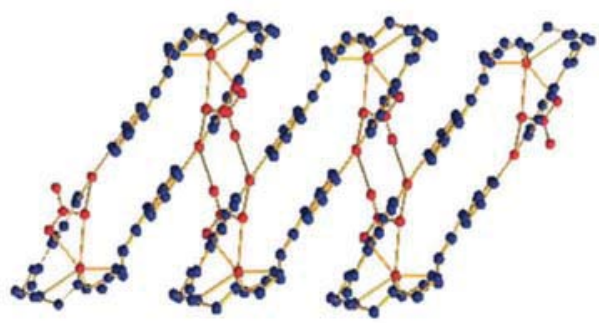

b)
Figure 3. (a) Isolated rings and (b) connected ring motifs obtained with Ln4. ${ }^{[9]}$

\section{Solubility Effects}

It should be mentioned here that the silver coordination compounds in general dissociate upon exposure to solvents, yielding smaller, often solvated, complexes in solution. For polymers, mixtures of short oligomers and/ or even monomers with coordinated solvent molecules can be found in the mass spectra. Since we have recently reviewed compounds with Li1-Li2 and Ln1-Ln2 


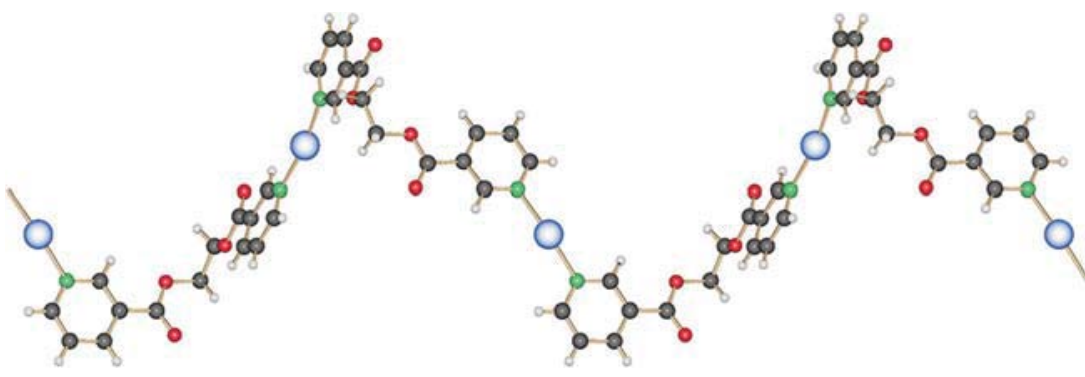

Figure 4. Helical 1D polymer obtained with $\operatorname{Ln} 1$ (color codes: $\mathrm{Ag}=$ blue; $\mathrm{N}=$ green; $\mathrm{O}=$ red; $\mathrm{C}=$ grey, $\mathrm{H}=$ white); anions omitted for clarity.

a)

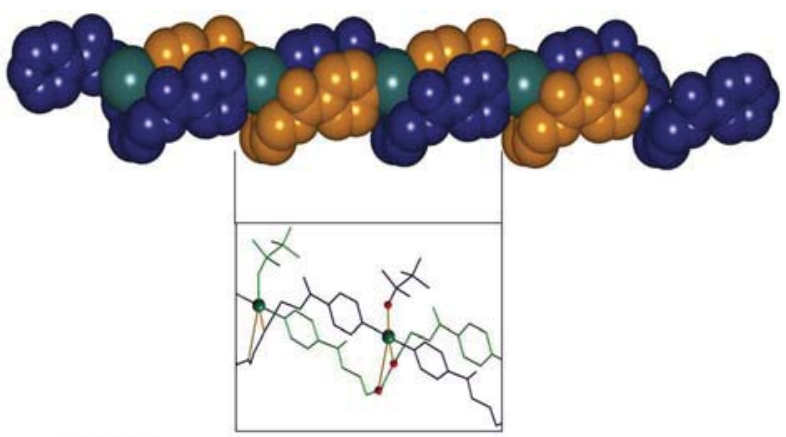

b)
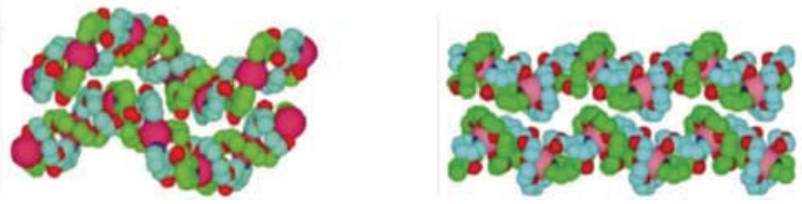

Figure 5. (a) Double helix formation upon coordination of $\mathrm{Li} 3$ to $\mathrm{Ag}$ $\left(\mathrm{CF}_{3} \mathrm{SO}_{3}\right)$, in which the anion coordinates to the metal ion (inlet); $\mathrm{H}$ atoms omitted for clarity. ${ }^{[9]}$ (b) Double helix formation with Ln4 and $\mathrm{AgBF}_{4}$ and (c) with Ln6 and $\mathrm{AgPF}_{6}$ as found by Hosseini et al. ${ }^{[21]}$

in terms of their structures and solubilities, a resumé on the general solubility trends for aqueous media will be given here using relative solubilities. In general, the zero-dimensional ring-type compounds are more soluble than the coordination polymers. Hence more silver is released into the environment from such metallacyclic compounds. From the polymeric structures, simple chains can form, for example, 'monomeric' complexes of the composition $[\mathrm{L}-\mathrm{Ag}-\mathrm{L}]^{+}$with different dipole moments. ${ }^{[9]}$ We have found that one-dimensional chains with such polar complexes are more soluble than apolar ones (Scheme 4). Helical single-stranded polymers are also more soluble than the simple chains with apolar units due to the polarity of the building blocks and the higher solubility of the ligand itself. We observed that, although heavily entangled, double helices are quite soluble, probably due to the fact that (i) the ligands become more soluble the longer they are and (ii) once a first ligand is dissolved, the silver ions become unsaturated and are more easily solvated.

In short, the apolar coordination polymer with the least polar structure has the lowest solubility. The solubility in aqueous media increases as the ligands become longer, independent of the complexity of the structure..$^{[9,14-19]}$

Compared to simple $\mathrm{AgNO}_{3}$, our compounds are generally less readily soluble, such as to tune the release of silver ions into the environment, thus gaining better control over the silver ion concentration, which should fall into the therapeutic window.

\section{Antimicrobial Properties and Biocompatibility}

The fact that silver ions possess good antimicrobial properties against most bacteria, viruses and fungi is probably because silver ions do not have a preferred target in those cells. ${ }^{[6]}$ Rather, they interact with a larger number of biomolecules, starting with the membrane and its components, via enzymes to DNA. It is said that they bind to soft as well as hard donor atoms, namely sulfur, nitrogen and oxygen, and can take different coordination numbers and geometries. A detailed description of the interaction of silver with biomolecules can be found in our recent review. ${ }^{[6]}$

The scope of synthesizing silver coordination compounds is to use them in the context of antimicrobial properties, in particular for the protection of implants from implant infection. We have thus shown previously that it is possible to attach some of our compounds to the surface of metallic substrates. ${ }^{[23-27]}$ These coated surfaces can be fully characterized, such that the nature of the coating is very well known. With these coated samples, in vitro measurements were made to determine the antibacterial properties as well as biocompatibility. ${ }^{[23,27]}$ In order to gain insight into the mechanism of action of our compounds, we also exposed Escherichia coli bacteria to silver and investigated upand down-regulated genes. ${ }^{[26]}$ We propose interaction of silver ions with Fe clusters, for example, releasing iron ions from the latter, thus generating high amounts of radical oxygen species (ROS). In general, the antibacterial action of our compounds can be correlated with the relative release of silver by the solid compounds into agar or broth solutions, and hence with the solubility of the compounds. We tested all ligands alone and found them to have no effect. Hence it is really the released silver ions which are responsible for the antibacterial properties. Our compounds are more active against Staphylococcus epidermidis than against S. aureus, which is in line with what others describe and may be due to the fact that $S$. aureus is more virulent. ${ }^{[23-27]}$ The general trend is that the more a compound is soluble, and hence releases silver ions, the more active it is against these two bacterial strains - relative to the amount of silver per mass unit of the respective compound. ${ }^{[9]}$ The highest solubility is observed for the ring compounds, with the most silver ions released per formula unit for the short ligand L2i. ${ }^{[24,25]}$ Such compounds would be good coatings for any surface to be protected from bacterial colonization. However, such a coating would also be consumed quite rapidly due to the ready solubility of the compounds and hence would be useful for short-term protection. ${ }^{[27]}$ It is recommended that the light-stable version of such metallacycles be used in this context, to avoid silver nanoparticle formation - an effect which would slow down the silver release dramatically. ${ }^{[6,7]}$ Following the ring-type compounds, the helical compounds are more soluble and hence more effective against both bacterial strains than the chain compounds with shorter ligands, despite the fact that they release less silver per formula unit.

While good antimicrobial activity is certainly required for many applications, in hospitals or the hygienic sector, for example, the use of silver compounds inside the body is limited due to the therapeutic window above which silver compounds are not only antimicrobial, but also cytotoxic to our own cells. We have discussed the current state of the literature in a recent review, and restrict ourselves to our own previous studies. ${ }^{[6]}$ In vitro tests 
with fibroblast cells have shown that, in general, cells tolerate the least soluble compounds to a greater extent than the more soluble compounds. ${ }^{[27]}$ For these low-soluble chain compounds based on Li1, a therapeutic window was observed, while for compounds with ligands L2 and higher the amount of released silver is cytotoxic even at low concentrations, yielding only a small or even no therapeutic window at all. For the compounds based on Li1, initial in vivo tests using a mouse model showed good biocompatibility towards leukocytes which, after a first reduction of viability to $80 \%$ after 2 days, recover completely after 5 days to full viability. ${ }^{[26]}$

\section{Outlook}

In order to further elucidate the molecular mechanism of action, we have started to investigate the interaction of silver ions with amino acids ${ }^{[28]}$ and peptides. ${ }^{[29,30]}$ Furthermore, we are developing new silver-based compounds with other ligands in order to enlarge our pool of compounds to be tested as antimicrobial agents. $^{[31-34]}$

\section{Conclusions}

Coordination compounds of silver with (iso-)nicotinic acid disubstituted oligoethylene oxide based ligands can be made in the form of metallacycles, simple chain compounds, and single and double helices. Depending on the ligands and the counter ions, these compounds possess different light sensitivity and solubility, both of which can be well controlled and tuned. This control is important as it influences the application potential in the area of biology and medicine.

\section{References}

[1] K. M. Fromm, Nature Chem. 2011, 3, 178

[2] For example, J. Y. Chen, Pediatr. Infect. Dis. J. 1992, 11, 1026.

[3] For example, F. W. Fuller, J. Burn Care Res. 2009, 30, 464.

[4] For example, J. R. Morones-Ramirez, J. A. Winkler, C. S. Spina, J. J. Collins, Sci. Transl. Med. 2013, 5, 190ra81, and references therein.

[5] a) For example, W. K. Jung, H. C. Koo, K. W. Kim, S. Shin, S. H. Kim, Y. H. Park, Appl. Environ. Microbiol. 2008, 74, 2171;

b) M. Ip, S. L. Lui, V. K. M. Poon, I. Lung, A. Burd, J. Med. Microbiol. 2006, 55, 59;

c) A. B. G. Lansdown, in Biofunctional Textiles and the Skin, (Eds: U.-C. Hipler, P. Elsner), Karger, Basel, 2006, p. 17.
[6] S. Eckhardt, P. S. Brunetto, J. Gagnon, M. Priebe, B. Giese, K. M. Fromm, Chem. Rev. 2013, 113, 4708.

[7] M. J. Hajipour, K. M. Fromm, A. AkbarAshkarran, D. J. de Aberasturi, I. R. de Larramendi, T. Rojo, V. Serpooshan, W. J. Parak, M. Mahmoudi, Trends Biotechnol. 2012, 30, 499.

[8] A. Y. Robin, K. M. Fromm, Coord. Chem. Rev. 2006, 250, 2127.

[9] I. Chevrier, J. Sagué, P. S. Brunetto, N. Khanna, Z. Rajacic, K. M. Fromm, Dalton Trans. 2013, 42, 217.

[10] J. L. Sagué, T. Vig Slenters, P. S. Brunetto, S. Zuber, A. Fleury, L. Mirolo, A. Y. Robin, M. Meuwly, O. Gordon, R. Landmann, A. U. Daniels, K. M. Fromm, Materials 2010, 3, 3407.

[11] T. Vig Slenters, Novel silver containing antimicrobial coatings for implant materials: new applications of $\mathrm{Ag}(\mathrm{I})$ coordination networks, PhD thesis, University of Basel, 2008.

[12] J. L. Sagué Doimeadios, A. Y. Robin, K. M. Fromm, Chem. Commun. 2005, 36, 4548 .

[13] J. L. Sague, K. M. Fromm, Cryst. Growth Des. 2006, 6, 1566.

[14] K. M. Fromm, A. Y. Robin, M. Meuwly, H. Goesmann, G. Bernardinelli, CrystEngComm 2004, 6, 336.

[15] K. M. Fromm, E. D. Gueneau, A. Y. Robin, W. Maudez, J. Sague, R. Bergougnant, Z. Anorg. Allg. Chem. 2005, 631, 1725.

[16] A. Y. Robin, J. L. Sagué, K. M. Fromm, CrystEngComm 2006, 8, 403.

[17] A. Y. Robin, J. L. Sague Doimeadios, A. Neels, T. Vig Slenters, K. M. Fromm, Inorg. Chim. Acta 2007, 360, 212.

[18] J. L. Sague, M. Meuwly, K. M. Fromm, CrystEngComm 2008, 10, 1542.

[19] K. M. Fromm, J. L. Sagué, L. Mirolo, Macromol. Symp. 2010, 291-292, 75.

[20] J. L. Doimeadios Sagué, Silver coordination compounds with a family of ditopic ligands of varying flexibility: about chains, rings, helices and polycatenanes, PhD thesis, University of Basel, 2006.

[21] B. Schmaltz, A. Jouaiti, M. W. Hosseini, A. De Cian, Chem. Commun. 2001, 1242.

[22] J. Girard, Development of new silver metallopolymers for biomaterials, PhD thesis, University of Fribourg, 2012.

[23] T. Vig Slenters, I. Hauser-Gerspach, A. U. Daniels, K. M. Fromm, J. Mat. Chem. 2008, 18, 5359.

[24] C. R. Arciola, N. Balaban, L. Baldassarri, K. Fromm, G. M. Hansch, U. Obst, E. Presterl, S. Stefani, J. Verran, L. Visai, Int. J. Artif. Organs 2008, 31, 858.

[25] K. M. Fromm, P. Brunetto, T. Vig Slenters, Abstracts of Papers, 237th ACS National Meeting, Salt Lake City, UT, 22-26 March 2009.

[26] O. Gordon, T. Vig Slenters, P. S. Brunetto, A. E. Villaruz, D. E. Sturdevant, M. Otto, R. Landmann, K. M. Fromm, Antimicrob. Agents Chemother. 2010, 54, 4208.

[27] P. S. Brunetto, T. Vig Slenters, K. M. Fromm, Materials 2011, 4, 355.

[28] L. Mirolo, T. Schmidt, S. Eckhardt, M. Meuwly, K. M. Fromm, Chem. Eur. J. 2013, 19, 1754.

[29] K. Belser, T. Vig Slenters, C. Pfumbidzai, G. Upert, L. Mirolo, K. M. Fromm, H. Wennemers, Angew. Chem. Int. Ed. 2009, 48, 3661.

[30] A. Mantion, A. G. Guex, A. Foelske, L. Mirolo, K. M. Fromm, M. Painsi, A. Taubert, Soft Matter 2008, 4, 606.

[31] F. Gschwind, A. Crochet, W. Maudez, K. M. Fromm, Chimia 2010, 64, 299.

[32] J. Chen, A. Neels, K. M. Fromm, Chem. Comm. 2010, 46, 8282.

[33] F. Gschwind, K. M. Fromm, CrystEngComm 2012, 14, 4008.

[34] J. Girard, K. M. Fromm, CrystEngComm 2012, 14, 6487. 